Ann. Génét. Sél. anim., x969, 1 (4), 447-463.

\title{
BIBLIOGRAPHIE SIGNALÉTITQUE
}

\section{INTERSEXUALITÉ ASSOCIÉE A L'ABSENCE DE CORNES CHEZ LA CHEVRE D'ORIGINE ALPINE}

\author{
Denise BOYAJEAN \\ Station centrale de Génétique animale, \\ Centre national de Recherches zootechniques, 78 -Jouy-en-Josas \\ Institut national de la Recherche agronomique
}

\section{INTRODUCTION}

Dans la présente bibliographie signalétique nous avons rassemblé les articles concernant l'hérédité des troubles sexuels et de l'absence de cornes chez la Chèvre domestique. Ces deux caractères sont constamment associés dans des populations caprines d'origine alpine utilisées comme amélioratrices dans le monde entier.

La présentation dans un graphique, lustre par lustre, du nombre des articles (fig. 1) permet d'entrevoir l'intérêt porté à ce sujet dans le passé.

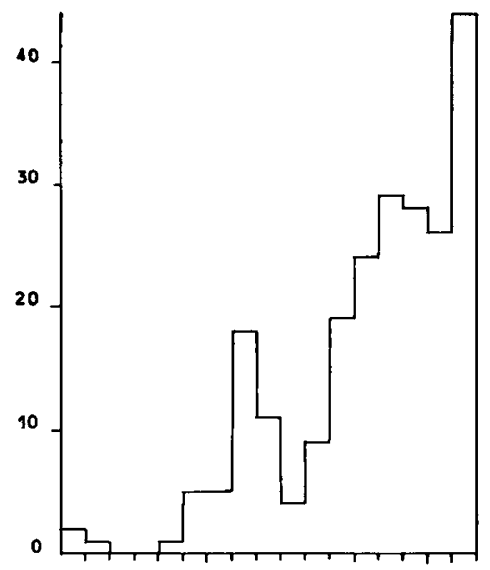

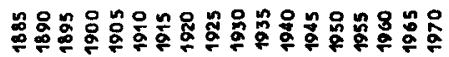

Frg. I. - Nombre d'articles consacrés à l'intersexualité liée à l'absence de cornes, lustrēpar lustre. 
En examinant ce diagramme on peut distinguer deux périodes : des origines à l'année 1935 et de l'année 1935 à nos jours.

Par la localisation géographique des travaux (fig. 2) et par les sujets abordés (fig. 3), les deux périodes se caractérisent assez bien : au cours de la première période, les auteurs sont surtout des Allemands et les études sont de type descriptif; au cours de la seconde période, la dis-

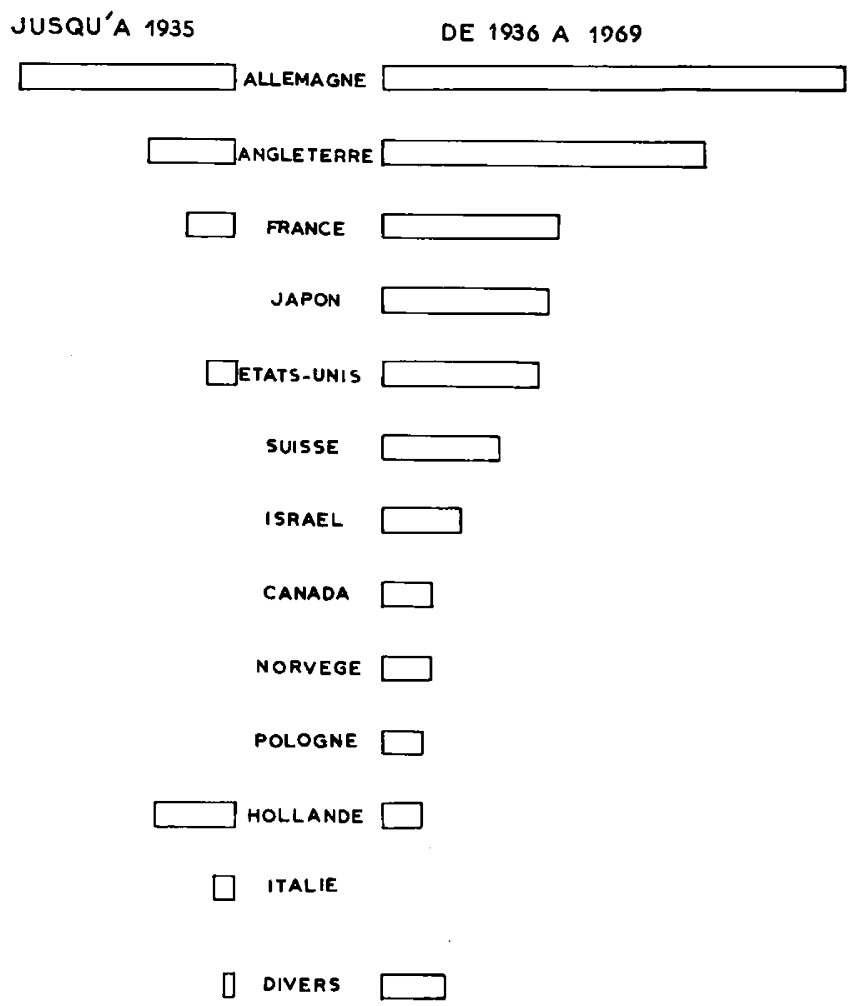

FIG. 2. - Répartition géographique des articles consacrés à l'intersexualité au cours des deux périodes : jus$q u ' a ̀ ~ r 935$ et de 1936 à 1969

JUSQU'A 1935

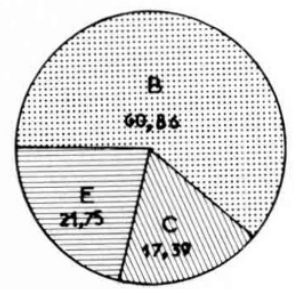

DE 1936 A 1969

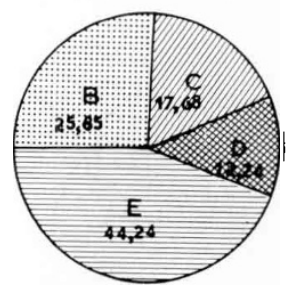

FIG. 3. - Répartition en pourcentage des sujets abordés par les articles consacrés à l'intersexualité associée $\dot{a}$ l'absence de cornes, au cours des deux périodes : jusqu'à 1935 et de 1936 à nos jours.

$\mathrm{B}$ : Intersexualité - Etude descriptive.

C : Stérilité des boucs - Etude descriptive.

$\mathrm{D}$ : Déterminations cytologiques et caryologiques du sexe.

$\mathrm{E}:$ Etudes génétiques. 
persion géographique des chercheurs est beaucoup plus grande et ces derniers embiassent un plus large éventail de sujets. Le ralentissement constaté pendant cette seconde période de 1955 à 1965 est certainement dû à l'insuffisance des techniques cytogénétiques (cf. Hulot, BasRur $n^{\circ}$ 217). Or, la détermination génétique du sexe, qui n'est possible que grâce à ces techniques, est en effet indispensable dans l'étude d'un tel phénomène.

Quant à la forme des documents originaux on doit noter une forte proportion de thèses (en Allemagne) et la présence de lettres à l'éditeur (en Angleterre). Ce dernier mode d'expression propice à l'éclosion des idées neuves est assez délaissé de nos jours par les chercheurs pour qu'on le remarque. Il est parfaitement illustré par le débat qui s'est instauré de 1942 à 1946 , dans les colonnes de Monthly Journal of British Goat Society (n ${ }^{\circ 8} 65,69,74,78,79,80,81,84$, $85,88,89,91$ ), en l'occurrence journal professionnel sans prétention scientifique. Au bout de ce débat une nouvelle conception génétique a été émise par AsDell (cf. Lauvergne no 218).

Les articles sont présentés chronologiquement, avec un ordre alphabétique d'auteurs à l'intérieur de chaque année. Ils sont répertoriés dans un index matières et un index auteurs. Chaque référence est suivie d'une ou plusieurs lettres qui renvoient à l'index matières.

\title{
I. - LISTE CHRONOLOGIQUE DES ARTICIES
}

\author{
1888
}

1. Morot C., 1888. Dilatation congénitale de la partie extra-pelvienne antérieure du canal de l'urètre chez les chevreaux mâles. Bull. Soc. cent. Méd. vét., 42, 431-434. B 2.

1889

2. Morot C., 1889. Fissure urétrale inférieure congénitale chez trois chevreaux mâles. Bull. Soc. cent. Méd. vét., 43, 37-39. B 2 .

3. Petit G., 1894. Nouvelle observation d'hermaphrodisme complexe des voies génitales, chez un bouc. Recl. Méd. vét., Ec. Alfort, 71, 247-249. B 2.

1903

4. Bourdelle M.-E., 1903. Hermaphrodisme complexe des voies génitales et apparent masculin chez un bouc. Revue vét., Toulouse, 11, 682-688. B2.

1909

5. Hink, 1909. Die Ursachen der Unfruchtbarkeit bei Ziegenböcken und Giesen. Z. Ziegenzucht., 232. C.

6. Eggers J., 1910. Untersuchungen über in den letzten Jahren ZahIreich beobachtete Fälle von Befruchtungsunfähigkeit bei Ziegenböcken. Med. vet. Diss., Leipzig, 19 p. C - I.

7. Kronacher C., 1910. Einiges über die biologischen Verhältnisse der Ziegen unter besonderer Berücksichtigung der Ursachen der Unfruchtbarkeit. Z. Ziegenz., 193-209. C.

8. Kronacher C., 1910. Einiges über die biologischen Verhältnisse der Ziegen unter besonderer Berücksichtigung der Ursachen der Unfruchtbarkeit. Dt. landwirt. Tierzucht, 14, (voir no 7).

1912

9. Davies C.-J., 1912. Heredity in goats. Mendel J., (sept.), E 1.

Annales de Génétique et de Sélection animale. - r97o. 
10. Davies C.-J., 1913. Caprine freemartins. Vet. J., 20, 62-70. B 11.

1915

11. Cutore, 1915. Pseudohermaphrodisme mâle chez un chevreau. Archs ital. Biol., 34, B 2.

12. Wester J., 1915. Unfruchtbarkeit bei Böcken. Dt. tierärztl. Wschr., 23, 451-458. C.

13. Wester J., 1915. Unfruchtbarkeit bei Böcken. Tijdschr. Veeartsenijk. Veeteelt, 42, C.

1918

14. Krediet G., 1918. Un cas d'hermaphrodisme vrai chez la chèvre (en néerlandais). Tijdschr. Diergeneesk., 45, 223-235. B 2.

15. Krediet G., 1919. On hermaphrodism in Goat (en néerlandais). Tijdschr. Diergeneesk., 46, 1-26. $H 2$.

16. Bartlitz P., 1920. Mikroskopische Untersuchungen der Nebenhoden unfruchtbarer Ziegenböcke. Med.-vet. Diss., Dresde. C.

17. Krediet G., 1920. Un deuxième cas d" "Hermaphroditismus verus bilateralis " chez la Chèvre; note préliminaire (en néerlandais). Tijdschr. Diergeneesk., 47, 134-139. $B 2$.

18. Richter J., Götze R., 1920. Weitere Untersuchungen über die Unfruchtbarkeit der Ziegenböcke. Berl. Tierärztl. Wschr., 36, 381-383. C.

\section{1}

19. ВАсн K., 1921. Pseudohermaphrodismus masculinus completus bei einer Ziege. Med.-vet. Diss., Berlin, 16 p. $B 2-I$.

20. Bruhlmeyer G., 1921. Pseudohermaphroditismus masculinus completus bei einer Ziege. Med.-vet. Diss., Berlin. B $2-I$.

21. Krediet G., 1921. Ovariotestes bei der Ziege. Biol. Zbl., 41, 447-455. B 2.

22. Rehfeldt R., 1921. Ein Fall von Pseudohermaphroditismus masculinus bei der Ziege. Med.-vet. Diss., Berlin, 31 p. $B 2-I$.

\section{2}

23. Beduhn F., 1922. Ein Fall von Pseudohermaphroditismus masculinus bei der Ziege. Med.vet. Diss., Berlin. $B 2-I$.

24. Krediet G., 1922. Eine Untersuchung der Geschlechtsdrüsen von dreissig neugeborenen Ziegen. Ein Fall von wahrem unilateralen Hermaphroditismus. Anat. Anz., 55, 502-510. $B$ 2.

25. KREdiet G., 1922. Un examen des gonades chez 30 chèvres nouveaux-nés (en néerlandais). Tijdschr. Diergeneesk., 49, 287-292. B 2 .

\section{3}

26. Berger H., 1923. Ein Fall von Pseudohermaphroditismus masculinus bei der Ziege. Med.vet. Diss., Berlin. B $2-I$.

27. Crew F.-A.-E., 1923. Studies in intersexuality. I. A peculiar type of developmental intersexuality in the male of the domesticated mammals. Proc. R. Soc. G. B., 95, 90-109. $B 2$.

28. Crew F.-A.-E., 1923. Developmental intersexuality in the goat. Yb. Br. Goat Soc., 6164. $B 2$.

29. Crew F.-A.-E., Dighton A., 1923. Developmental intersexuality in the domestic goat. Vet. J., 79, 135-139. B 2 .

30. Prange F., 1923. Vier Fälle von zygotischer Intersexualität bei der Hausziege. Zool. $J b ., 40,187-214 . B 2$. 
31. Töllner W., 1923. Pseudohermaphroditismus bei der Ziege. Med.-vet. Diss., Berlin, 9 p. $B 2-I$.

32. Howland, 1924. Relative sex frequency of issue from pure Toggenburg goats. Goat World, 9, 3-4. E 5 .

33. Töllner W., 1924. Pseudohermaphroditismus bei der Ziege. Arch. wiss. prakt. Tierheilk., 50, 205-213 (voir n'31).

34. Asdell S.-A., Crew F.-A.-E., 1925. The inheritance of horns in the goat. J. Genet., 15, 367-374. $E 1$.

35. Hagenstein F., 1925. Pseudohermaphroditismus masculinus bei der Ziege. Med.-vet, Diss., Berlin. $B 2-I$.

36. Rosenfeld R., 1925. Pseudohermaphroditismus masculinus und Hypospadia perinealis beim Ziegenbock. Med.-vet. Diss., Berlin, 14 p. $B 2-I$.

37. Scholz K., 1925. Ein Fall von Pseudohermaphroditismus masculinus (Hermaphroditismus tubularis interna) bei der Ziege. Med.-vet. Diss., Berlin, 12 p. $B 2-I$.

\section{6}

38. LusH J.-L., 1926. Inheritance of horns, wattles and color in grade Toggenburg goats. $J$. Hered., 17, 73-91. E 1 .

39. Niemann F., 1926. Ein Fall von Hypospadia interscrotalis beim Ziegenbock. Med.-vet. Diss., Berlin, 16 p. $B 2-I$.

\section{7}

40. Krediet G., 1927. Über die Genese der Ovariotestes. Wilhelm Roux' Arch. EntwMech., 109, 390-410. B 2 .

41. Asdell S.-A., Buchanan Smith A.-D., 1928. Inheritance of color, beard, tassels and horns in the Goat. J. Hered., 19, 425-430.'E 1.

42. Giuliani R., 1928. Esperienze di genetica nelle capre. Costituzione di una famiglia "senza corna " e con "attitudine ai parti bigemini e trigemini ". Riv. Zootec., 4, (11), 1-13. E 1.

1929

43. Krediet G., 1929. Intersexualität oder Hermaphroditismus bei Säugetieren. Z. Anat. EntwGesch., 91, 251-291. B 2-G.

44. Savage A., 1929. A note on a congenitally sterile Billy goat. Sci. Agr., 7, 463. C.

$$
1930
$$

45. Podhradsky J., 1930. A contribution to the knowledge of causes of the sterility in the bucks (en tchèque). Bull. Ec. sup. Agron., Brno, Rčs, 17, 27 p. C.

\section{1}

46. Richten J., 1931. Die Fruchtbarkeit der Ziege und ihre züchterische Auswertung. Züchtungskunde, 6, 401-416. E 5.

47. Funkouist H., 1932. Über Vererbung von Hypospadie bei Ziegen. Biologia Gen., 8, 59-64. $E$ 2.

\section{4}

48. Asdell S.-A., 1934. Goat research. Yb. Br. Goat Soc., 68-70. (Anim. Breed. Abstr., 1934, 2, 219). $E 1$. 
49. Addington L.-H., Cunningham O.-C., 1935. Milk goat breeding. New Mex. Agric. Exp. Stn Bull. (229), 89 p. (Horns and wattles, 49-58). (Anim. Breed. Abstr., 1935, 3, 392). E 1.

50. Asdell S.-A., 1935. Goat research in 1934. Yb. Br. Goat. Soc., 101, 103-104. (Anim. Breed. Abstr., 1935, 3, 393). B 2 .

\section{6}

51. Asdell S.-A., 1936. Hermaphroditism in Goats. Dairy Goat J., 14, 3-4.

52. Boring A.-M., Kao Y., 1936. Two hermaphrodite goats. Peking nat. Hist. Bull., 11, 115 118. (Anim. Breed. Abstr., 1938, 6). B 2 .

1937

53. OTx F., 1937. Über die erbliche Bedingtheit der Unfruchtbarkeit der Ziegenlammböcke. Züchtungskunde. 12, 460-468. E 2 .

54. Somervall M.-L., 1937. Intersexual development in the goat. Vet. J., 93, 60-61. (Anim. Breed. Abstr., 1939, 7). B 2.

1939

55. Eaton O.-N.; Simmons V.-L., 1939. Hermaphrodism in milk goats. J. Hered., 30, 261266. (Anim. Breed. Abstr., 1940, 8, 53). E 2.

56. Мовсом A.-R., 1939. Horned or hornless? Yb. Br. Goat Soc., 66, 63-67. E 1.

57. Navez O., 1939. Huit cas d'hermaphrodisme interne chez la chèvre. Annls Méd. vét., 84, 1-17. $B 2$.

1940

58. Degode F., 1940. Beobachtung und Untersuchung einer Anzahl von Ziegenintersexen. Jena Z. Natur W., 73, 145-230. B 2 .

\section{1}

59. Bennet-Clark T.-A., 1941. The inheritance of horns. Mon. J. Br. Goat Soc., 34, $202-205$. E 1.

60. Hill R.-T., 1941. Abnormal sex anatomy of a goat. Endocrinology, 29, 1003-1007. B 2. 61. Masuda S., SaEkr Y., 1941. On the intersex of goats (en japonais). Nogyo oyobi Eugei, 16, 1907-1911. $B 2-E 4$.

62. Rosenberger G., 1941. Die Unfruchtbarkeit der jungen Ziegenböcke und Massnahmen zur Verhütung der durch sie bedingten wirtschaftlichen Schäden. Dt. tierärztl. Wschr., 49, 449-454. $C$.

63. Asdell S.-A., 1942. Goat research in 1941. Yb. Br. Goat Soc., 50-51. E 1.

64. Asdell S.-A., 1942. The accessory reproductive tract in mammalian true hermaphrodites, an effect of position. Am. Nat., 76, 75-84. B 2 .

65. Holmes W.-F., 1942. Lettre à l'éditeur. Mon. J. Br. Goat Soc., 35, 122. E $1-H 1$.

66. Krediet G., 1942. Intersexuality in mammals. J. Noorderyn Zn., Gorinchem. (Anim. Breed. Abstr., 1947, 15, 205).

\section{3}

67. Eaton O.-N., 1943. An anatomical study of Hermaphrodism in Goats. Am. J. vet. Res., 4, 333-343. $B 2$.

68. Oppermann T., 1943. Zur Frage hornlose und gehörnte Böcke. Norddt. Schäferzeitung, 35, 115. C.

69. Paget R.-F., 1943. Lettre à l'éditeur. Mon. J. Br. Goat Soc., 36, 17 . (Anim. Breed. Abstr., 1943, 11, 238). E $4-H 1$

70. Paget R.-F., 1943. Hermaphrodites. Mon. J. Br. Goat Soc., 36, 57-59. (Anim. Breed. Abstr., 1943, 11, 238). E 4. 
1944

71. Anonyme, 1944. Hornlessness and intersexuality in goats. J. Hered., 35, 39. $E$ 4-H 2 (résumé de no 72).

72. Asdell S.-A., 1944. Hermaphrodites. Mon. J. Br. Goat Soc., 37, 13-15. (Anim. Breed. Abstr., 1944, 12, 88). E 4 (réponse à $\mathrm{n}^{\circ}$ 70)

73. Aspell S.-A., 1944. The genetic sex of intersexual goats and a probable linkage with the gene for hornlessness. Science, N.-Y., 99, 124. (Anim. Breed. Abstr., 1944, 12, 88). E 4.

74. Egerton J.-R., 1944. Lettre à l'éditeur. Mon. J. Br. Goat Soc., 37, (déc.), 2 (suppl. Br. Goat Soc. off. Not.) $E \&-H 1$.

75. Folley S.-J., 1944. The problem of hermaphrodism. Yb. Br. Goat Soc., 11-14. (Anim. Breed. Abstr., 1944, 12, 144). E 2.

76. Jeffery H.-E., 1944. The british Saanen. Yb. Br. Goat Soc., 4-9. $E$.

\section{5}

77. Eaton O.-N., 1945. The relation between polled and hermaphroditic characters in dairy goat. Genetics, Princeton, 30, 51-61. (Anim. Breed. Abstr., 1945, 13, 93-94). E 4.

78. Holmes W.-F., 1945. Lettre à l'éditeur. Mon. J. Br. Goat Soc., 38, $17 . \quad E 4-H 1$.

79. Holmes W.-F., 1945. Lettre à l'éditeur. Mon. J. Br. Goat. Soc., 38, (feb.), 2-4, (suppl. Br. Goat Soc., off. Not.). (Anim. Breed. Abstr., 1945, 18, 94). E $4-H 1$

80. Kitching J.-W,, 1945. Lettre à l'éditeur. Mon. J. Br. Goat. Soc., 38, $17 . E 4-H 1$.

81. Knapp J.-B., 1945. Lettre à l'éditeur. Mon. J. Br. Goat Soc., 38, 17. E $4-H 1$.

82. Ottaway C.-W., 1945. A case of intersexuality in a horned goat. Mon. J. Br. Goat Soc., 38, 61. B 12.

83. Ottaway C.-W., Arnold R.-H., 1945. A case of intersexuality (hermaphrodism) in a horned goat. Vet. J., 101, 150-153. (Anim. Breed. Abstr., 1946, 14, 29). B $12-E 4$.

84. Paget R.-F., 1945. Sex, intersex, sex-linkage and milk yield in goats. Mon. J. Br. Goat Soc., 38, 25-28. $E$ 2- $H 1$.

85. Paget R.-F., 1945. Lettre à l'éditeur. Mon. J. Br. Goat Soc., 38, (feb.), 2 (suppl. Br. Goat Soc. off. Not.). $E 4-H 1$.

1946

86. Aspell S.-A., 1946. Goat research in 1944-45. Yb. Br. Goat Soc., 29-32. (Anim. Breed. Abstr., 1946, 14, 156). E 4.

87. Eaton O.-N., 1946. Physiology of reproduction. 5. How to identify hermaphrodism. Better Goatkeeping, (déc.). $B$ 11.

88. Hellyer G.-C., 1946. Lettre à l'éditeur. Mon. J. Br. Goat Soc., 39, 6-7. E 4 - H 1 .

89. Hellyer G.-C., 1946. Lettre à l'éditeur. Mon. J. Br. Goat Soc., 39, 124-126. (Anim. Breed. Abstr., 1946, 14, 237). E $4-H 1$.

90. Miller A.-W., 1946. Report of the chief of the Bureau of animal industry. Agr. Res. Admin. Berl. Münch. Tierärztl. Wschr., 59, 60.

91. Paget R.-F., 1946. Hornless goats. Mon. J. Br. Goat Soc., 39, 80. (Anim. Breed. Abstr., $1946,14,156) . E 4-H 1$.

92. Paget R.-F., 1946. Heads and horns. Mon. J. Br. Goat Soc., 39, 187-189. (Anim. Breed. Abstr., 1947, 15, 38). E 1 .

1947

93. Eaton O.-N., 1947. Physiology of reproduction. 6. The inheritance of hermaphrodism. Better Goatkeeping, (jan.). E 2.

94. O’Mara J.-G., 1947. Hermaphroditism in the Goat. Dairy goat J., 25, 2-7.

1948

95. Kondo K., 1948. Genetic studies on the intersex in milk goats. I. An anatomical study of intersexual goats (en japonais, rés. anglais) Jap. J. zootech. Sci., 19, 1 - 4. (Anim. Breed. Abstr., 1951, 19, 217). B 2 .

96. Masur K., Kondo K., 1948. On the intersex in milk goats. Jap. J. Genet, 23, 24-25. (Anim. Breed. Abstr., 1951, 19, 361). B 2.

97. Moen O., 1948. Hermaphrodisme (intersexualité) chez la chèvre (en norvégien). Bonde og Smăbruker. 
98. JefFery H.-E., 1949. Examine that kid. Yb. Br. Goat Soc., 51-52. B 11.

99. Moen O., 1949. Chèvres intersexuées (en norvégien). Sau Geit, 3, 44. B 2.

100. Nieberle K., Сohr P., 1949. Lehrbuch der spezial pathologische Anatomie der Haustiere. G. Fischer, Iena. $B 2$.

\section{0}

101. Makino S., 1950. Constitution of the sex chromosome in an intersex goat. Pap. Coord. com. Res. Genet., 1, 1-3. (Anim. Breed. Abstr., 1953, 814) (Jap. Sci. Rev. Biol., 1952, 1, no 830). D.

102. Masui K., Kondo K., 1950. Studies on intersexuality in milkgoat (en japonais). Jap. J. Genet., 25, 54. E 5 .

103. Rako A., 1950. Beitrag zur Kenntnis der Leistungseigenschaften der Kreuzungsprodukte zwischen einheimischen Ziegen und Saanenziegen. Schweizer. Arch. Tierheilk., 92, 305-320, E 4 .

\section{1}

104. Herm G., 1951. Innere Zwitter und die Möglichkeit ihrer Feststellung. Ziegenzüchter, 42, 160. B 2 .

105. Honeker A., 1951. Hornlosigkeit und Stummelhornbildung beim Ziegenbock. Berl. Münch. Tierärztl. Wschr., 64, 138-139. (Anim. Breed. Abstr., 1952, 20, 156). A.

106. Honeker A., König R., 1951. Wie zeigt sich die Zwitterbildung bei Ziegenlämmern ? Milchgebende Ziegenböcke. Ziegenzüchter, 42, 60. B 2.

107. Kone ker A., König R., 1951. Die Zwitterbildung und deren Erkennung beim Ziegenlamm. In : die Krankheiten der Ziege, (2), die Krankheiten des Geschlechtapparates der Ziege. Verlag für Kleintierzucht, Dortmund. $B 2-G$.

108. Косн W., 1951. Die Diagnose der Sterilität bei Ziegen. Tierärzll. Umsch., 6, 153-155. (Anim. Breed. Abstr., 1952, 20, 157). C.

109. König R., 1951. Die Krankheiten der männlichen Ziege. Die Unfruchtbarkeit der Ziegenböcke. Die künstliche Besamung in der Ziegenzucht. In : die Krankheiten der Ziege, (2), die Krankheiten des Geschlechtsapparates der Ziege. Verlag für Kleintierzucht, Dortmund, 11-25. $C-G$.

\section{2}

110. Berge S., 1952. Chèvres " intersexuées " sans cornes. Norsk. Landbr., 6, $96 . \quad B 12$.

111. Bilgemre K., Düzgünes O., 1952. Hornlessness in Angora Goat. Z. Tierzücht ZüchtBiol., 60, 282-284. (Anim. Breed. Abstr., 1953, 21, 164). E $1-F$.

112. Kondo K., 1952. Studies on intersexuality in milk goats. Jap. J. Genet., 27, $131-141$. (Anim. Breed. Abstr., 1953, 21, 277). B $11-E 2-E 4$.

113. Kondo K., 1952. Frequency of occurence of intersexes in milk goats (abstr. en japonais). Jap. J. Genet., 27, 227. (Anim. Breed. Abstr., 1953, 21, 304). E 5.

114. Kondo K., 1952. Genetic studies on the intersex in milk goats. II. Genetic sex of intersexual goats (en japonais, rés. anglais). Pap. coord. Com. Res. Genet., 3, 161-166. (Anim. Breed. Abstr., 1956, 24, 167). (Jap. Sci. Rev. Biol., 1954, 644). E $2-E$ 5.

115. Löliger H.C., 1952. Bericht über die bisherigen Ergebnisse der Erforschung der Samenstauung bei Ziegenböcken. Kleintierzüchter, (22). $C-G$.

116. NaIto, M. 1952. Studies on the anterior lobe of pituitary in the intersexual goats (en japonais rés. anglais). Jap. J. zootech. Sci., 23, 56-67. (Anim. Breed. Abstr., 1954., 22, 135). $B 2$.

117. Simic V.N., 1952. Cas rarissime d'intersexualité chez la Chèvre (en serbe). Acta vet. Beogr., 2, 147-159. B 2.

118. SprehN C., 1952. Zwitterbildung und Hornlosigkeit bei Ziegen. Dt. Kleintierzüchter, 8 (4). B 12.

1953

119. Aspell S.A., 1953. Factors involved in sterility of farm animals. Iowa St. Coll. J. Sci., 28, 127-132. E 4 .

120. DrvekaR K.V., 1953. A case of hermaphrodism in a horned milk goat. Indian vet. $J$., 29, 538-540. (Anim. Breed. Abstr., 1954, 22, 53). B 12.

121. Hallex G., Baxter J.S., 1953. Leucocyte invasion of the genital tract of an intersexual goat. J. comp. Path., 63, 179-183. (Anim. Breed. Abstr., 1953, 21, 369). B 11 
122. Löliger H.C., 1953. Beitrag zum Problem der Samenstauung bei Ziegenböcken. Fortpfl. Besam. Haustiere, 23, 6-7, 12. C.

123. Naito M., 1953. Studies on the anterior lobe of hypophysis in the intersexual goats. $Z$. Tierzücht ZüchtBiol., 61, 201-252. (Anim. Breed. Abstr., 1953, 21, 369). B 2.

124. Schanz R., 1953. Missbildungen und ihre Genese in der West-Berliner Ziegenzucht. Lämmerjahrgang 1952. Med.-vet. Diss., Berlin. $B 11-B 2-I$.

125. Simic V.N., 1953. Un autre cas intéressant d'intersexualité chez la Chèvre (en serbe). Acta vet. Beogr., 3, 201-212. (Recl Méd. vét. Ec. Alfort, 1953, 130, 513). B 11.

126. Sprehn K., Löliger H.C., 1953. Ueber die verschiedenen Ursachen der Unfruchtbarkeit bei Ziegenböcken. Kleintierzucht Forsch. lehre, 2, 243-246. (Anim. Breed. Abstr., 1956, 24, 271). $C$

127. Werner C., 1953. Histologische Untersuchungun über die Samenstauung bei Ziegenböcken. Med.-vet. Diss., Berlin. C.

\section{4}

128. Schönherr S., 1954. Die Unfruchtbarkeit der Ziegenböcke, ihre Verbreitung, frühzeitige Erkennung und Bekämpfung. Med.-vet. Diss., Berlin, 163 p. $C-I$.

129. Sснӧnherr S., 1954. Sterilität bei Ziegen und ihre volkswirtschaftliche Bedeutung. Berl. Münch. Tierärztl. Wschr., 67, 311-313. (Anim. Breed. Abstr., 1955, 23, 398). C.

\section{5}

130. Anonyme, 1955. Die bisherige und zukünftige Erforschung der Unfruchtbarkeit der Ziegenböcke. Ziegenzüchter, 5, (mars). $C-H 2$.

131. Honeker A., 1955. Die bisherige und zukünftige Erforschung der Unfruchtbarkeit der Ziegenböcke. Ziegenzüchter, 45, 49-53. C.

132. Kondo K., 1955. The frequency of occurence of intersexes in milkgoats. Jap. J. Genet., 30, 139-146. (Anim. Breed. Abstr., 1956, 24, 167). E 5.

133. Kondo K., 1955. Studies on intersexuality in milk goats. III. On the homozygous recessive males and the methods to eliminate the intersexual gene (en japonais, rés. anglais). Bull. natn Inst. agri. Sci., Tokyo G, 10, 117-123. (Anim. Breed. Abstr., 1955, 23, 396-397). E 4.

134. Jamdar M.N., 1955. A case of true hermaphroditism in a goat. Indian vet. $J$., 32, 230232. (Anim. Breed. Abstr., 1956, 24, 166). B 2.

135. Löhle K., HerrmanN, 1955. Unfruchtbarkeitsuntersuchungen bei den zur Körung aufgetriebenen Ziegenböcken in Thüringen. $D t$ landwirt., 6, $42 . \quad C$.

\section{6}

136. HoNeker A., 1956. Ziegenbockkörungen in Baden-Württemberg. Tierärztl. Umsch. 10, 373-375. C.

137. Löliger H.C., 1956. Unfruchtbarkeit bei Ziegenböcken und ihr Auftreten in der Schweiz. Kleintierzucht Forsch. lehre, 5, 161-166. (Anim. Breed. Abstr., 1958, 26, 299). C.

138. Löliger H.C., 1956. Unfruchtbarkeit bei Ziegenböcken und ihr Auftreten in der Schweiz. Kleinviehzüchter, 4, 576-580, 594-595 (voir no 137).

139. Löliger H.C., 1956. Der Keimgehalt des Ziegenbockspermas. Fortpfl. Züchthyg. Haustierbesam., 6, 77-78. C.

140. Nakahara T., 1956. Studies on the sex chromatin in cell nuclei of the goat. I. Detection of the sex chromatin in nerve cells (en japonais). Jap. J. anim. Reprod., 2, 69-72. D.

141. Schönherr S., 1956. Die Unfruchtbarkeit der Ziegenböcke, ihre Verbreitung, frühzeitige Erkennung und Bekämpfung. Z. Tierzücht. ZüchtBiol., 66, 209-234, 381-415 (Anim. Breed. Abstr., 1956, 24, 270-271) (voir no 128).

142. Schumann H., 1956. Hornlosigkeit, ihre Vererbung und ihre Folgen in der Ziegenzucht. Tierzüchter, 8, 296-301. (Anim. Breed. Abstr., 1956, 24, 377). E 4.

\section{7}

143. BuEchi H.F., 1957. Untersuchungen über das verschobene Geschlechtsverhältnis, die Intersexualität und die Fruchtbarkeit bei der Milchziege. Z. Tierzücht. ZüchtBiol., 69, 3090. (Anim. Breed. Abstr., 1957, 25, 285). E 5.

144. Koсh P., Fischer H., Schumann H., 1957. Erbpathologie der landwirtschaftlichen Haustiere. P. Parey, Berlin, 436 p., 257-258, 265-271. (Anim. Breed. Abstr., 1957, 25, 438-439). $E 1-E 2-E 4-H 2$. 
145. Löliger H.C., 1957. Die Patogenese der sogennant Samenstauung in Nebenhoden der Ziegenböcke. Z Zbl. vet. Med., 4, 892-906. C.

146. Löliger H.C., 1957. Die Verbreitung von Unfruchtbarkeit unter den männlichen Tieren verschiedener Ziegenrassen in deutschen und ausländischen Zuchtgebieten. Zuchthyg. Fortpflstör. Besam. Haustiere, 1, 201-215. (Anim. Breed. Abstr., 1960, 28, 159). C.

147. Roslanowski K., Lukasik J., 1957. Hermaphrodism in goats. I. Frequency of occurence (en polonais). Medycyna wet., 13, 603-605. (Anim. Breed. Abstr., 1958, 26, 300). E 5 .

148. Widmaier R., 1957. Untersuchungen an intersexuellen Ziegenlammern im Hinblick auf die Unfruchtbarkeit der Bucke. Wiss. Z. Martin-Luther - Univ. Halle-Wittenb., 6, 67-96. B 11 .

\section{8}

149. Brandsch H., 1958. Die Vererbung geschlechticher Anomalien und des Hornes bei der Hausziege in ihrer gegenseitigen Beziehung - Versuch einer genetischen Analyse. Kühn Archiv., 72, 449-450. E 4 .

150. Crepin P., 1958. Les cornes dans l'espèce caprine. Mouton, 13, 79. (Anim. Breed. Abstr., 1958, 28, 412). F.

151. Vrtel M., Kudla ̌̆ E., Veznik Z., 1958. Hypoplasy and atrophy of the testicles in Hegoats (en tchèque). Vet. Med., 31, 627-640. C.

152. Widmaier R., 1958. Histologische Untersuchungen an Holden von Ziegenböckchen und hodenähnlichen Gonaden von Ziegenintersexen. Wiss. Z. Martin-Luther-Univ. Halle-Wittenb., 8, 55-73. $C$.

153. Altenkirch W., Brandsch H., 1959. Kreuzungsversuche mit gehörnten Ziegen zur Klärung der Frage der Intersexualität und der Unfruchtbarkeit bei Ziegenböcken. Arch. Geflügelz. Kleintierk., 8, 363-380. (Anim. Breed. Abstr., 1961, 29, 185). E 4.

154. Blockнuis J., 1959. Kunstmatige inseminatie bij geiten in de provincie Utrecht. Tijdschr. Diergeneesk., 84, 347-351. (Anim. Breed. Abstr., 1959, 27, 317). E 5.

155. Brandsch H., 1959. Die Vererbung geschlechtlicher Missbildung und des Hornes bei der Hausziege in ihrer gegenseitigen Beziehung. Versuch einer genetischen Analyse. Arch. Geflügelz. Kleintierk., 8, 310-362. (Anim. Breed. Abstr., 1961, 29, 185-186). E 4 - E 5.

156. Brandsch H., 1959. Beitrag zum Problem des gestörten Geschlechtsverhältnisses bei Ziegen. Wiss. Z. Martin-Luther-Univ. Halle-Wittenb. Math.-naturw. Reihe, 8, 571-576. (Anim. Breed. Abstr., 1961, 29, 186). E $4-E 5$.

157. Weber W., 1959. Erbliche Grundlagen von Fruchtbarkeit und Fruchtbarkeitsstörungen. Schweiz. Landw. Mh., 37, 425-435 (Anim. Breed. Abstr., 1960, 28, 14). C.

158. Weber W., 1959. Erbliche Grundlagen von Fruchtbarkeit und Fruchtbarkeitsstörungen. Schr. Schweiz. Verein. Tierz. (25) 17-27 (Anim. Breed. Abstr., 1960, 28, 376) (voir n⿳157).

159. Widmaier R., 1959. Über die Beziehung zwischen den sogenannten Konkrementen in den Hodenkanälchen und heterosexuellen Missbildungen der Hodenkanälchen. Biol. Zbl., 78, 799-812. (Anim. Breed. Abstr., 1960, 28, 292). C.

\section{0}

160. Bielańska-Osuchowska Z., 1960. Hermaphroditism in Goat. III. Cytogenetic sex diagnosis in hermaphrodite goats (en polonais). Medycyna wet., 16, 658-662. (Anim. Breed. Abstr., 1961, 29, 314). D.

161. Bielańska-Osuchowska Z., Roslanowski K., 1960. Hermaphroditism in Goats. II. Developmental malformations of the reproductive organs (en polonais). Medycyna wet., 16, 599-602. (Anim. Breed. Abstr., 1961, 29, 314). B 2.

162. Bielańska-Osuchowska Z., Roslanowski K., 1960 . Developmental malformations of the reproductive organs in Goats (en polonais). Roczn. Naukro rln, E, 70, 327-330. (Anim. Breed. Abstr., 1961, 29, 448-449). B 2.

163. Brandsch H., Erdmann S., 1960. Kernmorphologische Untersuchungen zur Geschlechtserkennung bei Ziegen. Arch. Geflügelz. Kleintierk., 9, 174-181. D.

164. Haugen E., 1960. Hermaphroditism in Goats (en norvégien). Meld. Norg. LandbrHoisk., 39, (10), 1-33. B $11-E 4-E 5$.

165. Jong S. dE, 1960. Is there a relationship between the horned condition in goats and the production of intersexes male infertility and disturbed sex ratio (en néerlandais). Geitehouder, 23, 40-41. (Anim. Breed. Abstr., 1961, 29, 314-315). E $4-H 2$. 
166. Luers T., Struck E., 1960. Untersuchungen zur geschlechtspezifischen Struktur der Neutrophilenkerne bei einigen Haustieren (Ziege, Schaf, Schwein) unter Berücksichtigung der Zwitter. Zool. Anz., 164, 89-103. (Anim. Breed. Abstr., 1960, 28, 242). D.

\section{1}

167. Löhle K., Barfuss E., 1961. Untersuchungen über Korpergewichte und Körpermasse bei fruchtbaren und unfruchtbaren Ziegenböcken. Arch. Geflugelz. Kleintierk., 10, 121-128. C.

168. WEBER W., 1961. Erfahrungen über die Sterilitätsuntersuchungen bei Ziegenböcken. Kleinviehzüchter, (22), 511-515. C.

169. Laor M., Barnea R., Angel Huguette, Soller M., 1962. Polledness and hermaphroditism in Saanen goats. Israel J. agric. Res., 12, 83-88. E 4.

170. Makino S., Sasaki T., Sofuni, 1962. Chromosomes of domestic mammals with sex anomalies. Chromosome Inf. serv., 3, 29-30. D.

171. Mc Entee K., 1962. Pathology of the female. In Joest E. Handbuch der speziellen pathologischen Anatomie der Haustiere, 3 Aufl., 4, 130-201 (Chèvres intersexuées, 144-145). $B 2-H 2$.

172. Nes N., Andersen K., Slagsvold P., 1963. Chromosomes des chèvres hermaphrodites (en norvégien). Medlemsbl. Norske Veterinaerfor., (7), 155-166. $D$.

173. OnUma H., Nishikawa Y., 1963. The sex chromatin of domestic animals. III. On the genetic sex of bovine freemartin and goat intersex (en japonais, rés. anglais). Bull. natn. Inst. Anim. Ind. Jap., (2), 277-286. D.

174. Ricordeau G., 1963. Possibilités de sélection dans l'espèce caprine. Bull. tech. Inf. Ingrs. Servs. agric., (179), 189-204. E 5 - H 2.

175. Soller M., Avgel Huguette, Laor M., Barnea R., Ayalon N., Weiss Y., 1963. Polledness and abnormal sex-ratios, male infertility and litter size in Saanen dairy goats. $X I^{\mathrm{e}}$ Congr. int. Génét., sept. 1963, La Haye, 1 p. (non publié). $E 3-E 4$.

176. Soller M., Laor M., Barnea R., Weiss Y., Ayalon N., 1963. Polledness and infertility in male Saanen goats. J. Hered., 54, 237-240. E 4.

1964

177. Basrur Parvathi K., Coubrough R.I., 1964. Anatomical and cytological sex of a Saanen Goat. Cytogenetics, 3, 414-426. $B 2$ - D.

178. Beatty R.A., 1964. Chromosome deviations and sex in vertebrates. In Armstrong C.N. and Marshall A.J., ed., Intersexuality in Vertebrates including man, Academic Press, London, 17-133. (Chromosomes deviations and sex in Vertebrates, 115). H 2.

179. Bonfert A., 1964. Expériences dans l'insémination des chèvres avec du sperme congelé Elevage Insem., (83), 3-6. E 5 .

180. BRAndsch., 1964. Vergleichende Untersuchungen zur Vererbung des Kryptorchismus und der Intersexualität bei Haustieren. $E$ 4. (Anim. Breed. Abstr., 1965, 21, 30).

181. Laor M., Barnea R., Angel Huguette, Ayalon N., Weiss Y., Soller M., 1964. Polledness and hermaphroditism male sterility, sex-ratios and litter size in Israël Saanen Goats. Int. Goat Breeders Conf., Jul. 1964, London, 6 p. (ronéoté). $E 3-E 4-E 5$.

182. Sionneau G., 1964. Étude tératologique de la Chèvre domestique. Thèse Méd. vét., Alfort, 59 p. $B 2-H 2$ - I.

183. Soller M., ANGel Huguette, 1964. Polledness and abnormal sex ratio in Saanen goats. J. Hered., 55, 139-142. E $4-E 5$.

184. Soller M., Kempenich O., 1964. Polledness and litter size in Saanen goats. J. Hered., 55, 301-304. E 3 .

185. Tegtmeyer M., 1964. Hérédité des défauts sexuels et troubles fonctionnels chez la Chèvre. Chève, 7, (34) 10-11. G. 
186. Grouchy J. de, Lauvergne J.J., Ricordeau G., 1965. Etudes cytogénétiques chez 16 chèvres intersexuées. C. R. hebd. Séanc. Acad. Sci., Paris, 260, 2932-2935, $D$.

187. Padeh B., Wysoki M., Ayalon N., Soller M., 1965. An XX/XY hermaphrodite in the goat. Israel J. med. Sci., 1, 1008-1012. (Excerpta Medica, 1966, 4, 305). B $12-D$.

188. Tegtmeyer M., 1965. Hérédité des défauts sexuels et troubles fonctionnels chez la Chèvre Chèvre, 8, (35), 14-17. G. (suite de no 185).

1966

189. Anonyme, 1966. Fortpflanzungsstörungen bei hornlosen Ziegenrassen nach Berichten und Mitteilungen aus Israel, der Schweiz und den U.S.A. ADZ Mitt., 2, (3), 10-12. $H 2$. 190. Alder S., 1966. Ziegenbesamung in der Schweiz. Kleintierzüchter, 14, 346-351. E 5. 191. Biggers J.D., McFeely R.A., 1966. Intersexuality in domestic mammals. Adv. Reprod. Physiol., 1, 29-60 (Aberrations of genetic and chromosomal origin, 32, 36-39). $D$.

192. Hafez E.S.E., Jainudeen M.R., 1966. Intersexuality in farm animals. Anim. Breed. A bstr., 34, 1-15. G.

193. Mооде K.L., 1966. The sex chromatin of freemartins and other animal intersexes. In Moore K.L. ed., the sex chromatin, W.B. Saunders Company, Philadelphia, 229-240 (Sex chromatin and intersexuality in goats, 235). H 2.

194. Tegtmeyer M., 1966. Über die Vererbung geschlechtlicher Missbildungen und Funktionsstörungen bei Ziegen. ADZ-Mitt., 2 (3), 12-16. G.

\section{7}

195. Bomsel-Helmreich O., Thibault C., 1967. Anomalies chromosomiques et troubles pathologiques ou sexuels chez les Mammifères. Annls Biol., 6, 287-322. H 2.

196. Hulot Françoise, Lauvergne J.J., 1967. Les chromosomes des ruminants. Annls Génét., 10, 86-97. $\mathrm{H}_{2}$.

197. Ilbery P.L.T., Williams D., 1967. Evidence of the freemartin condition in the goat. Cytogenetics, 6, 276-285. B 11, D.

198. Mc Feely R.A., Hare W.C.D., Biggers J.D., 1967. Chromosome studies in 14 cases of intersex in domestic animals. Cytogenetics, 6, 242-253. B 11, D.

199. Ricordeau G., Lauvergne J.J., 1967. Hypothèse génétique unique pour expliquer la présence d'intersexués, de mâles en excès et de mâles stériles en race caprine Saanen. Annls Zootech., 16, 323-334. (Anim. Breed. Abstr., 1969, 37, 604). E 3,E 4, E 5 .

200. Suzuki S., Hayashida S., Yamauchi C., Nozawa K., Tanaka K., Watanabe S., NishiNAKagawa H., Shotake 'T., 1967. Genetical studies on the native farm animals in Japan and her adjacent localities. 2. On native goats in South Western Islands (en japonais, rés. anglais). Jap. J. Zootech. Sci., 38, 452. E 4.

201. Tegtmeyer M., 1967. Abschluss des Referates über die Vererbung geschlechtlicher Missbildungen und Funktionsstörungen bei Ziegen von $\mathrm{Nr} 3$ (6) Dezember 1966 und Erklärung von Fremdwörtern und Fachausdrücken auch des bereits erschienenen Teiles. ADZ Mitt., 3, (1), 14-16. G.

202. Weber W., 1967. Unfruchtbarkeit bei Ziegenböcken. Kleinviehzüchter, (26), 899-901. C.

\section{8}

203. Anonyme, 1968. Le bouc est une chèvre. Chèvre, 11, (53), 30. H 2 .

204. Basrur Parvathi K., Kanagawa H., 1968. Cytogenetic studies on intersex goats (abstr.). Can. J. Genet. Cytol., 10, 764. D.

205. Biggers J.D., 1968. Aspects of intersexuality in domestic mammals. In Vle Congr. int. Reprod. anim. Insem. artif., juil. 1968, Paris, 2, 841-870. H 2.

206. Fatoux A., 1968. Mon bouc est cornu. Mes chevrettes seront-elles cornues ? Chèvre, (53), 1-4. $H_{2}$.

207. Hamerton J.L., 1968. Significance of sex chromosome derived heterochromatin. Nature, 219, 910-914. $E 4$.

208. Hoekstra P., Vos mas M.G., 1968. The relation between polledness and infertility in goats. A survey of literature (en néerlandais). Tijdschr. Diergeneesk., 93, 1059-1066. (Anim. Breed. Abstr., 1969, 37, 93). G.

209. Lojpa L., 1968. Der chromosomale,-klinische und Sektionsbefund bei einigen Fällen der Intersexualität von Ziegen und Rind. VIe Congr. int. Reprod. anim. Insém. artif., juil. 1968, Paris, 2, 901-903. D. 
210. Lyngset O., 1968. Studies on reproduction in the goat. V. Pathological conditions and malformations of the genital organs of the goat. Acta Vet. Scand., 9, 364-375. $B 2$.

211. Platten M.E., 1968. Horns and hermaphrodism. Yb. Br. Goat Soc., 77, E 4.

212. Short R.V., Hamerton J.L., Grieves S.A., Pollard C.E., 1968. An intersex goat with a bilaterally asymmetrical reproductive tract. $J$. Reprod. Fert., 16, 283-294. B $11, D$.

213. Winzenried H.U., Zerobin K., 1968. Die künstliche Besamung von Ziegen während der Deckperioden $1965 / 66$ und 1966/67. Kleinviehzüchter, 16, 10-15. E 5.

\section{9}

214. Basrur Parvathi K., 1969. Some thoughts on the association of polled trait and intersexuality in goats., Annls Génét. Sél. anim., 1, 439-446. D.

215. Basrur Parvathi K., Kanagawa H., 1969. Adrenal morphology and hydroxysteroid dehydrogenase enzymes in normal and intersex goats. Annls Génét. Sél. anim., 1, 423-438. B2.

216. Basrur Parvathi K., Kanagawa H., 1969. Anatomic and cytogenetic studies on 19 hornless goats with sexual disorders. Annls Génét. Sél. anim., 1, 349-378. B $11-D$.

217. Conteel J.M., Hulot Françoise, Courot M., Attal J., Philippon A., 1969. Examens morphologiques, caryologiques, physiologiques et pathologiques de boucs stériles sans cornes. Annls Génét. Sél. anim., 1, 341-348. B 11 - D.

218. Hamerton J.L., Dickson J.M., Pollard C.E., Grieves S.A., Short R.V., 1969. Genetic intersexuality in goats. $J$. Reprod. Fert., Intersexuality, Suppl., 7, 25-51. $B 11-D$.

219. Hulot Françoise, Basrur Parvathi K. 1969. La détermination du sexe chromosomique dans l'étude de l'intersexualité associée à l'absence de cornes chez la Chèvre d'origine alpine : revue. Annls Génét. Sél. anim., 1, 383-390. D-G.

220. Lauvergne J.J., 1969. Progrès des connaissances génétiques sur l'intersexualité associée à l'absence de cornes chez la Chèvre d'origine alpine. Annls Génét. Sél. anim., 1, 403-412. E $5-G$.

221. Jost A., Lauvergne J.J., 1969. Quelques réflexions à l'issue du séminaire sur l'intersexualité chez la chèvre sans cornes. Annls Génét. Sél. anim., 1, 465-468. H 2.

222. Lauvergne J.J., 1969. Fréquence à l'équilibre du gène d'origine alpine responsable de l'absence de cornes et de l'intersexualité dans des populations de chèvres. Annls Génét. Sél. anim., 1, 413-422. E 5 .

223. Ricordeau G., 1969. La recherche agronomique et la production caprine. Rev. fr. Agric., (26), 61-72 (Amélioration génétique, les aspects théoriques, 66-67). $H 2$.

224. Ricordeau G., 1969. Surprolificité des génotypes sans cornes dans les races caprines alpine saanen, alpine chamoisée et poitevine. Annls Génét. Sél. anim., 1, 391-395. $E_{3}$.

225. Ricordeau G., Bouillon J., 1969. Variations de l'âge d'apparition du cornage dans les races caprines alpine saanen, alpine chamoisée et poitevine. Annls Génét. sél. anim., 1, 397-401. A.

226. Ricordeau G., Lauvergne J.J., Bouillon J., 1969. Hérédité du cornage dans l'espèce caprine et conséquences pour la sélection. Chèvre, (55), 1-3. H 2 .

227. Soller M., Padeh B., Wysoki M., Ayalon N., 1969. Cytogenetics of Saanen Goats showing abnormal development of the reproductive tract associated with the dominant gene for polledness. Cytogenetics, 8, 51-67. B $11-D-E 4$.

228. Weber W., 1969. La stérilité des mâles dans les races caprines suisses sans cornes. Annls Génét. Sél. anim., 1, 379-382. C.

\section{II. - INDEX MATIÈRES}

$$
\text { A. - Cornage - Etude descriptive }
$$

$105-225$.

$$
\text { B. - Intersexualité - Etude descriptive }
$$

B I. Cornage précisé

B II. SANS CORNES

$10-87-98-112-121-124-125-148-164-197-198-212-216$

$-217-218-227$. 
B I2. AVEC CORNES

$$
82-83-110-118-120 .
$$

B 2. Cornage non précisé

$$
\begin{aligned}
& 1-2-3-4-11-14-17-19-20-21-22-23-24-25-26-27- \\
& 28-29-30-31-33-35-36-37-39-40-43-50-52-54-57- \\
& 58-60-61-64-67-95-96-99-100-104-106-107-116- \\
& 117-123-124-134-161-162-171-173-182-210-215 .
\end{aligned}
$$

\section{C. - Stérilité des boucs - Etude descriptive}

$5-6-7-8-12-13-16-18-44-45-62-68-108-109-115-122-126-$ $127-128-129-130-131-135-136-137-138-139-141-145-146-151-$ $152-157-158-159-167-168-202-228$.

D. - Déterminations cytologiques et caryologiques du sexe

$101-140-160-163-166-170-172-173-177-182-187-191-197-198-$ $204-209-212-214-216-217-218-219-227$.

$$
\text { E. - Etudes génétiques }
$$

E I. Cornage

$$
9-34-38-41-42-48-49-56-59-63-65-92-111-144 .
$$

E 2. Anomalies sexuelles : Intersexualité et stérilité des boucs

$$
47-53-55-75-84-93-112-114-144 .
$$

E 3. Anomalies sexuelles et action sur la prolificité

$$
175-181-183-199-224 .
$$

$\mathrm{E}$ 4. Cornage et anomalies sexuelles

$69-70-71-72-73-74-77-78-79-80-81-83-85-86-88-89$ - $91-103-112-119-133-142-144-149-153-155-156-164-$ $165-169-175-176-180-181-183-199-200-207-211-227$.

E 5. Fréquence des anormaux et sex-ratio

$$
\begin{aligned}
& 32-46-61-76-102-113-114-132-143-147-154-155-156 \\
& -163-173-179-181-183-190-199-213-220-222 .
\end{aligned}
$$

$$
\text { F. - Chèvres sans cornes d'origine non alpine }
$$

$111-150$.

$$
\text { G. - Revues Bibliographiques }
$$

$43-107-109-115-185-188$ - $192-194-201-208-219-220$. 
$\mathrm{H}$ I. Lettre à l'éditeur

$$
65-69-74-78-79-80-81-84-85-88-89-91 \text {. }
$$

$\mathrm{H}$ 2. Reprises et résumés d'article (s) - conférences.

$$
\begin{gathered}
15-71-130-144-163-171-174-178-182-189-193-195-196- \\
203-205-206-221-223-226 . \\
\text { I. }- \text { Thèses } \\
6-19-20-22-23-26-31-35-36-37-39-124-128-182 .
\end{gathered}
$$

\section{III. - INDEX AUTEURS}

A

Anonyme : $71-130-189-203$.

AdDINGTON L.H. : 49.

Alder S. : 190.

Alten KIRCH W. : 153.

ANDERSEN K. : 172.

ANGEL Huguette : 169 - 175 - 181 - 183.

ARNold R.H. : 83.

AsDeLL S.A. : $34-41$ - 48 - 50 - 51 - 63 - 64 $72-73-86-119$.

ATtal J. : 217.

AYALON N. : $175-176-181-187-227$.

B

BACH K. : 19.

BARFUSS E. : 167.

BARNÉA R. : $169-175-176$ - 181.

BARTLITZ P. : 16.

BASRUR Parvathi K. : $177-204-214-215$ 216 - 219.

BaXTer J.S. : 121.

BEATTY R.A. : 178.

BEDUHN F. : 23.

BenNet-Clark T.A. : 59.

BERGE S. : 110

BERGER H. : 26.

BiELAŃSKA - OSUchows Ka Z. : 160 - 161 - 162.

Biggers J.D. : 191 - 198 - 205.

BILGEMRE K. : 111.

BLOCKHUIS J. : 154.

BOMSEL-HELMREICH O. : 195.

BONFERT A. : 179.

Boring A.M. : 52.

BouILlon J. $: 225$ - 226.

BOURDELLE M.E. : 4.

BRANDSGH H. : 149 - 153 - 155 - 156 - 163 180.

BRUhlMeyer G. : 20.

BuCHANAN SMITh A.D. : 41.

BUECHI H.F. : 143.

\section{C}

COHR P. : 100.

CoRTEEL J.M. : 217.

Courot M. : 217.
Coubrough R.I. : 177.

CREPIN P. : 150.

CREW F.A.E. : $27-28-29-34$.

CUNNINGHAM O.C. : 49.

Cutore : 11.

\section{D}

Davies C.J. : 9 - 10.

DEGODE F. : 58 .

Dickson J.M. : 218.

DIVEKAR K.V. : 120.

DÜZGÜNES 0 . : 111.

\section{E}

EAton O.N. : $55-67-77-87-93$.

EGERTON J.R. : 74.

EGGERS J. : 6.

ERDMANN S. : 163.

$\mathbf{F}$

Fatoux A. : 206.

FISCHER $H .: 144$.

FoLLEY S.J. : 75.

FunkQuist H. : 47.

\section{G}

Gruliani R. : 42.

GöTze R. : 18.

GRIEVES S.A. : 212 - 218.

Grouchy J. de : 186.

$\mathbf{H}$

HAFEZ E.S.E. : 192.

HAgenstein F. : 35.

HAlley G. : 121

HAMERTON J.L. : 207 - $212-218$.

HARE W.C.D. : 198.

HaUgen E. : 164.

HAYASHIDA S. : 200. 
HeIM G. : 104.

Hellyer G.C. : $88-89$.

HerrmanN : 135.

Hill R.T. : 60.

HiNk : 5.

HoEkstra P. : 208.

Holmes W.F. : $65-78-79$.

HONE KER A. : $105-106-107-131$ - 136 .

HOWLAND : 32.

Hulot Françoise : 196 - 217 - 219.

I

ILBERy P.L.T. : 197.

\section{$\mathrm{J}$}

JAINUDEEN M.R. : 192.

JAMDAR M.N. : 134.

JEFFERY H.E. : $76-98$.

JoNg S. de : 165.

Jost A. : 221.

K

KAO Y. : 52.

Kanagawa H. : $204-216$.

KEMPENICH O. : 184.

Kitching J.W. : 80 .

KNAPP J.B. : 81.

Косн P. : 144.

Kосн W. : 108.

KoNDO K. : 95 - 96 - 102 - 112 - 113 - 114 132 - 133.

KöNIG R. : $106-107-109$.

Krediet G. : $14-15-17-21-24-25-40$ $43-66$.

Kronacher C. : 7 - 8 .

Kudlă̆ E. : 151 .

\section{L}

LAOR M. : 169 - 175 - 176 - 181.

LAuvergne J.J. : 186 - $196-199$ - 219 $220-221-222-226$.

LÖHLE K. : 135 - 167 .

LOJDA L. : 209.

Löliger H.C. : 115 - 122 - 126 - 137 - 138 $139-145-146$.

LUERS T. : 166.

LUKASIK J. : 147

LUSH J.L. : 38 .

LYNGSET O. : 210 .

\section{$\mathbf{M}$}

Makino S. : $101-170$.

Masuda $S$. : 61.

MASUI K. : $96-102$.

Mc Entee K. : 171.

Mc FEely R.A. : 191 - 198.

Miller A.W. : 90

MoEN O. : 97 - 99 .

MoORE K.L. : 193.

Morcom A.R. : 56 .

Morot C. : $1-2$
N

Naito M. : 116 - 123.

Nakahara T. : 140 .

Navez O. : 57.

NES N. : 172.

Nieberle K. : 100.

NiemanN F. : 39.

Nishikawa Y. : 173.

Nishinakagawa H. : 200.

Nozawa K. : 200 .

\section{$\mathrm{O}$}

O'Mara J.G. : 94.

ONUMA H. : 173.

OppermanN T. : 68.

OtTaway C.W. : $82-83$.

OTT F. : 53

\section{$\mathbf{P}$}

PADEH B. : 187 - 227.

PAget R.F. : $69-70-84-85-91-92$.

Petit G. : 3.

Philippon A. : 217.

Platten M.E. : 211.

Podhradsky J. : 45.

Pollard C.E. : 212 - 218.

Prange F. : 30 .

$\mathbf{R}$

RAKO A. : 103.

REHFELDT R. : 22.

Richter J. : 18 - 46 .

Ricordeau G. : 174 - 186 - 199 - 223 - 224 $225-226$.

Rosenberger G. : 62 .

Rosenfeld R. : 36 .

Roslanows ki K. : 147 - 161 - 162.

\section{$\mathbf{S}$}

SAEkI Y. : 61 .

SASAKI T. : 170 .

SAVAGE A. : 44 .

SchaNZ R. : 124.

SCHOLZ K. : 37.

SCHÖNHERR S. : 128 - 129 - 141.

SchUMANN H. : $142-144$.

SHORT R.V. : $212-218$.

SHOTAKe T. : 200.

Simic V.N. : 117 - 125.

Simmons V.L. : 55.

SIONNEAU G. : 182.

Slagsvold P.: 172

SOFUNI : 170 .

SOLLER M. : 169 - 175 - 176 - 181 - 183 - 184

- 187 - 227.

SomervaIL M.L. : 54.

SPREHN C. : 118.

SPREHN K. : 126.

Struck E. : 166.

SUzUKI S. : 200.

TANAKA K. : 200 .

TegtMeyer M. : 185 - $188-194-201$.

Thibault C. : 195.

Töllner W. : $31-33$. 


\section{V}

VEZNIK Z. : 151

Vos MAAS M.G. : 208.

VRTEL M. : 151.

W

Watanabe S. : 200.

WEBER W. : 157 - 158 - 168 - 202 - 228.

WeISS Y. : 175 - 176 - 181 .

WERNER C. : 127.

WESTER J. : 12 - 13 .
Widmaier R. : 148 - 152 - 159.

WILLIAMS D. : 197.

WINZENRIED H.U. : 213.

WYsokI M. : 187 - 227

\section{$\mathbf{Y}$}

YamaUchi C. : 200.

Z

ZEROBIN K. : 213.

\section{IV. - SOURCES}

Le fichier a été constitué dans un premier temps à partir du livre de Koch P., Fisher W., Schumann H. (n 144) et de l'article de Buechi H.F. (nº 143).

Il a été complété ensuite par le dépouillement des revues analytiques telles que Animal Breeding Abstracts, Excerpta Medica, série XXII, et par la consultation du fichier de l'École vétérinaire d'Alfort pour les références de thèses vétérinaires.

Reçu pour publication en janvier 1970.

\section{REMERCIEMENTS}

Nous tenons à remercier M. J.-J. LAUVERGNE pour l'aide et les conseils qu'il nous a apportés nécessaires à l'élaboration de cette bibliographie et tous les documentalistes et bibliothécaires qui nous ont envoyé les documents. 